\title{
On homeomorphic and diffeomorphic solutions of the Abel equation on the plane
}

\author{
by ZBigniew LeŚNIAK (Kraków)
}

Abstract. We consider the Abel equation

$$
\varphi[f(x)]=\varphi(x)+a
$$

on the plane $\mathbb{R}^{2}$, where $f$ is a free mapping (i.e. $f$ is an orientation preserving homeomorphism of the plane onto itself with no fixed points). We find all its homeomorphic and diffeomorphic solutions $\varphi$ having positive Jacobian. Moreover, we give some conditions which are equivalent to $f$ being conjugate to a translation.

The aim of this paper is to find all homeomorphic and diffeomorphic solutions with positive Jacobian of the Abel equation

$$
\varphi[f(x)]=\varphi(x)+a
$$

on the plane $\mathbb{R}^{2}$. We assume that $a \neq(0,0)$ and $f$ is an orientation preserving homeomorphism of the plane onto itself with no fixed points (such a homeomorphism will be called a free mapping). By a curve is meant a homeomorphic image of a straight line. A curve is said to be a line (an open line in [4]) if it is a closed set.

1. We note that the existence of homeomorphic solutions $\varphi$ of (1) is equivalent to $f$ being conjugate to the translation $T(x)=x+a$ (i.e. $f=$ $\varphi^{-1} \circ T \circ \varphi$, where $\varphi$ is a homeomorphism). S. Andrea [1] has proved that a free mapping $f$ is conjugate to a translation if and only if

(H) for all $x, y \in \mathbb{R}^{2}$ there exists a curve segment $C$ with endpoints $x, y$ such that $f^{n}[C] \rightarrow \infty$ as $n \rightarrow \mp \infty$, where $f^{n}$ is the $n$th iterate of $f$.

In the present paper we give some other conditions equivalent to $(\mathrm{H})$. We introduce the following conditions:

1991 Mathematics Subject Classification: Primary 39B10; Secondary 54H20, 26A18.

Key words and phrases: functional Abel equation, free mapping. 
(A) There exists a homeomorphism $\varphi$ of the plane onto itself satisfying (1).

$\left(\mathrm{A}^{\prime}\right)$ There exists a homeomorphism $\varphi$ of the plane into itself satisfying (1).

(B) There exists a line $K$ such that

$$
\begin{gathered}
K \cap f[K]=\emptyset, \\
U^{0} \cap f\left[U^{0}\right]=\emptyset, \\
\bigcup_{n \in \mathbb{Z}} f^{n}\left[U^{0}\right]=\mathbb{R}^{2},
\end{gathered}
$$

where $U^{0}:=M^{0} \cup f[K]$ and $M^{0}$ is the strip bounded by $K$ and $f[K]$. (See Fig. 1.)

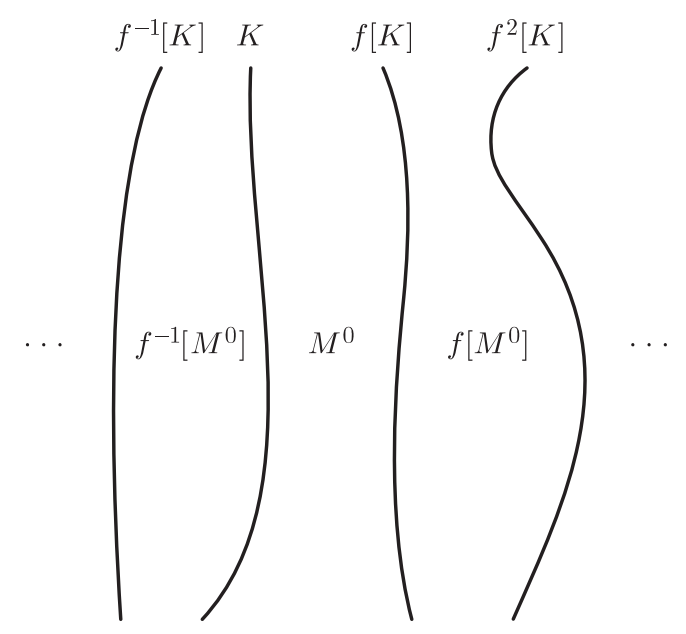

Fig. 1

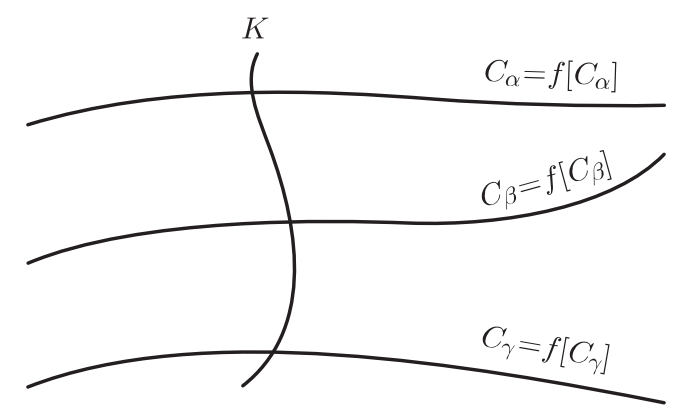

Fig. 2

(C) There exist a family of curves $\left\{C_{\alpha}: \alpha \in I\right\}$ and a line $K$ such that

$$
f\left[C_{\alpha}\right]=C_{\alpha} \quad \text { for } \alpha \in I,
$$




$$
\begin{gathered}
C_{\alpha} \cap C_{\beta}=\emptyset \quad \text { for } \alpha, \beta \in I, \alpha \neq \beta, \\
\operatorname{card}\left(K \cap C_{\alpha}\right)=1 \quad \text { for } \alpha \in I, \\
\bigcup_{\alpha \in I} C_{\alpha}=\mathbb{R}^{2} . \quad \text { (See Fig. 2.) }
\end{gathered}
$$

We shall show

Theorem 1. If $f$ is a free mapping, then conditions $(\mathrm{A}),\left(\mathrm{A}^{\prime}\right),(\mathrm{B})$ and (C) are equivalent.

2. First note the following

Lemma 1. Let $a=\left(a_{1}, a_{2}\right) \in \mathbb{R}^{2} \backslash\{(0,0)\}$ and $T(x):=x+a$ for $x \in \mathbb{R}^{2}$. Then there exists a homeomorphism $\psi$ of the plane onto itself such that

(9)

$$
T(x)=\psi^{-1}[\psi(x)+(1,0)] .
$$

Proof. Set

$$
\begin{array}{ll}
\psi\left(x_{1}, x_{2}\right)=\left(\frac{1}{a_{1}} x_{1},-\frac{a_{2}}{a_{1}} x_{1}+x_{2}\right) & \text { if } a_{1} \neq 0, \\
\psi\left(x_{1}, x_{2}\right)=\left(\frac{1}{a_{2}} x_{2}, x_{1}\right) & \text { if } a_{1}=0 .
\end{array}
$$

By Lemma 1, from now on we may assume that $a=(1,0)$.

Lemma 2. If $f$ is a free mapping, then ( $\left.\mathrm{A}^{\prime}\right)$ implies (B).

$\operatorname{Proof}$. Since $\varphi$ is a homeomorphism, $\varphi\left[\mathbb{R}^{2}\right]$ is a region. Moreover, $\varphi\left[\mathbb{R}^{2}\right]$ $=\varphi\left[\mathbb{R}^{2}\right]+(1,0)$. Put $T\left(x_{1}, x_{2}\right):=\left(x_{1}+1, x_{2}\right)$ and $T^{0}\left(x_{1}, x_{2}\right):=\left(x_{1}, x_{2}\right)$ for $\left(x_{1}, x_{2}\right) \in \varphi\left[\mathbb{R}^{2}\right]$. Write $L:=\left\{\left(x_{1}, x_{2}\right) \in \varphi\left[\mathbb{R}^{2}\right]: x_{1}=0\right\}$. Since $T^{n}[L]=$ $\left\{\left(x_{1}, x_{2}\right) \in \varphi\left[\mathbb{R}^{2}\right]: x_{1}=n\right\}$ for $n \in \mathbb{Z}$, we have

$$
T^{n}[L] \cap T^{m}[L]=\emptyset \quad \text { for } n, m \in \mathbb{Z}, n \neq m .
$$

Put $L^{n}:=T^{n}[L], N^{n}:=\left\{\left(x_{1}, x_{2}\right) \in \varphi\left[\mathbb{R}^{2}\right]: x_{1} \in(n, n+1)\right\}$ and $W^{n}:=$ $N^{n} \cup L^{n+1}$ for $n \in \mathbb{Z}$. Note that

$$
N^{n}=T^{n}\left[N^{0}\right] \quad \text { for } n \in \mathbb{Z},
$$

$$
W^{0} \cap W^{n}=\emptyset \quad \text { for } n \in \mathbb{Z} \backslash\{0\}, \quad \bigcup_{n \in \mathbb{Z}} W^{n}=\varphi\left[\mathbb{R}^{2}\right] .
$$

Put $K:=\varphi^{-1}[L]$ and $K^{n}:=f^{n}[K]$ for $n \in \mathbb{Z}$. By $\left(\mathrm{A}^{\prime}\right), \varphi \circ f^{n}=T^{n} \circ \varphi$, whence

$$
f^{n} \circ \varphi^{-1}=\varphi^{-1} \circ T^{n} .
$$

Hence

$$
K^{n}=f^{n}\left[\varphi^{-1}[L]\right]=\varphi^{-1}\left[T^{n}[L]\right]=\varphi^{-1}\left[L^{n}\right] .
$$

Therefore, by (10), $K^{n} \cap K^{m}=\emptyset$ for $n, m \in \mathbb{Z}, n \neq m$. 
Since $K=\varphi^{-1}[L]$ and $L$ is closed in $\varphi\left[\mathbb{R}^{2}\right]$, the curve $K$ is a line, and so is $K^{n}=f^{n}[K]$ for every $n \in \mathbb{Z}$.

For each $n \in \mathbb{Z}$, denote by $M^{n}$ the strip bounded by $K^{n}$ and $K^{n+1}$. Since $\varphi$ is a homeomorphism,

$$
M^{n}=\varphi^{-1}\left[N^{n}\right] \quad \text { for } n \in \mathbb{Z} .
$$

Hence by (11) we have

$$
f^{n}\left[M^{0}\right]=f^{n}\left[\varphi^{-1}\left[N^{0}\right]\right]=\varphi^{-1}\left[T^{n}\left[N^{0}\right]\right]=\varphi^{-1}\left[N^{n}\right]=M^{n}
$$

for $n \in \mathbb{Z}$.

Put $U^{n}:=M^{n} \cup K^{n+1}$ for $n \in \mathbb{Z}$. Then by (12) and (13),

$$
U^{n}=\varphi^{-1}\left[N^{n} \cup L^{n+1}\right]=\varphi^{-1}\left[W^{n}\right]
$$

and by (14),

$$
U^{n}=f^{n}\left[M^{0} \cup f[K]\right]=f^{n}\left[U^{0}\right] .
$$

Hence

and

$$
\bigcup_{n \in \mathbb{Z}} f^{n}\left[U^{0}\right]=\bigcup_{n \in \mathbb{Z}} U^{n}=\varphi^{-1}\left[\bigcup_{n \in \mathbb{Z}} W^{n}\right]=\mathbb{R}^{2}
$$

This completes the proof.

$$
f\left[U^{0}\right] \cap U^{0}=U^{1} \cap U^{0}=\varphi^{-1}\left[W^{1} \cap W^{0}\right]=\emptyset .
$$

THEOREM 2. Let $f$ be a free mapping of the plane onto itself and let $a=\left(a_{1}, a_{2}\right) \in \mathbb{R}^{2} \backslash\{(0,0)\}$. Assume that condition (B) is satisfied. Let $\varphi_{0}: U^{0} \cup K \rightarrow \mathbb{R}^{2}$ be continuous and suppose

$$
\varphi_{0}[f(x)]=\varphi_{0}(x)+a \quad \text { for } x \in K .
$$

Then:

(a) There exists a unique solution $\varphi$ of (1) such that

$$
\varphi(x)=\varphi_{0}(x) \quad \text { for } x \in U^{0} \cup K .
$$

This solution $\varphi$ is continuous.

(b) If $\varphi_{0}$ is one-to-one and $\varphi_{0}\left[U^{0}\right] \cap\left(\varphi_{0}\left[U^{0}\right]+k a\right)=\emptyset$ for all $k \in \mathbb{Z} \backslash\{0\}$ then $\varphi$ is a homeomorphism.

(c) If $\varphi_{0}$ is one-to-one, $\varphi_{0}[K]$ is a line and $\varphi_{0}[K] \cap D_{\gamma} \neq \emptyset$ for all $\gamma \in \mathbb{R}$, where $D_{\gamma}=\left\{\left(x_{1}, x_{2}\right) \in \mathbb{R}^{2}: a_{2} x_{1}-a_{1} x_{2}=\gamma\right\}$, then $\varphi$ is a homeomorphism.

(d) If $\varphi_{0}$ is one-to-one, $\varphi_{0}[K]$ is a line, $\varphi_{0}[K] \cap D_{\gamma} \neq \emptyset$ for all $\gamma \in \mathbb{R}$, and $\varphi_{0}\left[\operatorname{int} U^{0}\right]=N^{0}$, where $N^{0}$ is the strip bounded by $\varphi_{0}[K]$ and $\varphi_{0}[K]+a$, then $\varphi$ is a homeomorphism of $\mathbb{R}^{2}$ onto itself. (See Fig. 3.)

Proof. Since $K \cap f[K]=\emptyset$,

$$
f^{n}[K] \cap f^{n+1}[K]=\emptyset \quad \text { for } n \in \mathbb{Z} .
$$




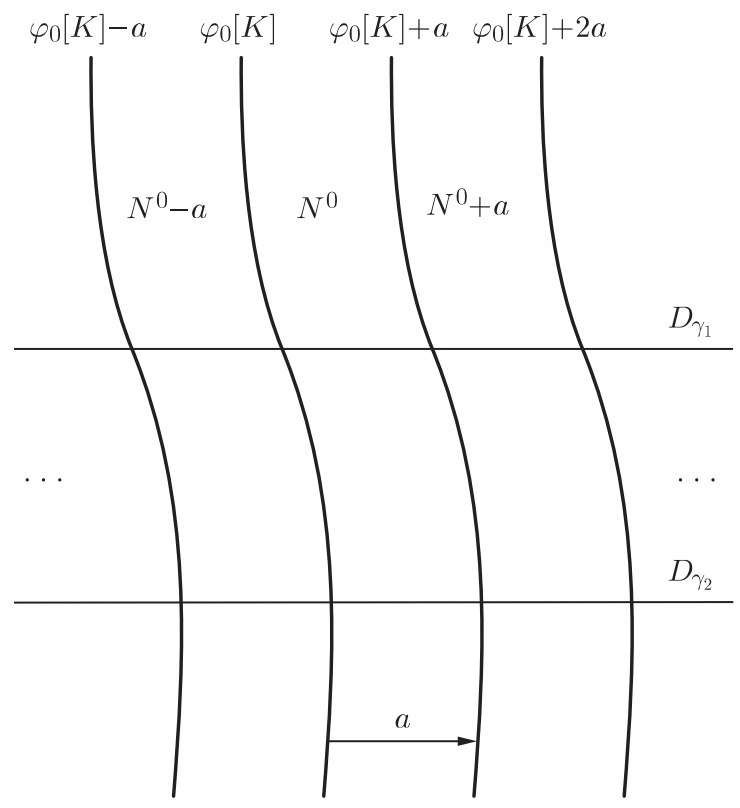

Fig. 3

Moreover, for each $n \in \mathbb{Z}$, the curve $f^{n}[K]$ is a line, since so is $K$. Denote by $M^{n}$ the strip bounded by $f^{n}[K]$ and $f^{n+1}[K]$. Let $U^{n}:=M^{n} \cup f^{n+1}[K]$ for $n \in \mathbb{Z}$. Since $f$ is a homeomorphism,

$$
U^{n}=f^{n}\left[U^{0}\right] \quad \text { for } n \in \mathbb{Z} .
$$

Furthermore, for every $n \in \mathbb{Z}, f^{n}[K]$ lies in the strip between $f^{n-1}[K]$ and $f^{n+1}[K], f^{n-1}[K] \cap M^{n}=\emptyset$ and $f^{n+1}[K] \cap M^{n-1}=\emptyset$. Otherwise we would have $f^{n-1}\left[U^{0}\right] \cap f^{n}\left[U^{0}\right] \neq \emptyset$, which contradicts (3).

Define $\varphi: \mathbb{R}^{2} \rightarrow \mathbb{R}^{2}$ by setting

$$
\varphi(x)=\varphi_{0}\left[f^{-k}(x)\right]+k a, \quad x \in U^{k}, k \in \mathbb{Z} .
$$

It is clear that $\varphi$ is a unique solution of (1) satisfying (16) and that $\varphi$ is continuous in $\bigcup_{n \in \mathbb{Z}}$ int $U^{n}$.

Take any $x_{0} \in K$. We now show that $\varphi$ is continuous at $x_{0}$. Let $P$ be a closed disc with centre at $x_{0}$ such that $P \cap f^{-1}[K] \neq \emptyset$ and $P \cap f[K] \neq \emptyset$. Then $P \cap f^{-1}[K]$ and $P \cap f[K]$ are compact. Let $R$ be an open disc with centre at $x_{0}$ and radius smaller than $\min \left\{\varrho\left(x_{0}, P \cap f^{-1}[K]\right), \varrho\left(x_{0}, P \cap f[K]\right)\right\}$, where $\varrho$ is the Euclidean metric on the plane. Then we have

$$
R \cap f^{-1}[K]=\emptyset \text { and } R \cap f[K]=\emptyset .
$$

Put $R_{1}:=R \cap \operatorname{int} U^{0}, R_{2}:=R \cap \operatorname{int} U^{-1}, R_{0}:=R \cap K$. Then $\varphi(x)=$ $\varphi_{0}(x)$ for $x \in R_{1}$, and $\varphi(x)=\varphi_{0}[f(x)]-a$ for $x \in R_{2} \cup R_{0}$. As $x_{0} \in R_{0}$ we hence get $\varphi\left(x_{0}\right)=\varphi_{0}\left(x_{0}\right)$ by $(15)$. 
Let $x_{k} \rightarrow x_{0}, x_{k} \in R$. If $x_{k} \in R_{1} \cup R_{0}$, then

$$
\lim _{k \rightarrow \infty} \varphi\left(x_{k}\right)=\lim _{k \rightarrow \infty} \varphi_{0}\left(x_{k}\right)=\varphi_{0}\left(x_{0}\right)=\varphi\left(x_{0}\right),
$$

because $\varphi_{0}$ is continuous in $R_{1} \cup R_{0} \subset K \cup U^{0}$. If $x_{k} \in R_{2}$, then $f\left(x_{k}\right) \in U^{0}$ and $f\left(x_{k}\right) \rightarrow f\left(x_{0}\right) \in U^{0}$. Thus

$$
\lim _{k \rightarrow \infty} \varphi\left(x_{k}\right)=\lim _{k \rightarrow \infty}\left(\varphi_{0}\left[f\left(x_{k}\right)\right]-a\right)=\varphi_{0}\left[f\left(x_{0}\right)\right]-a=\varphi_{0}\left(x_{0}\right)=\varphi\left(x_{0}\right),
$$

because $\varphi_{0}$ is continuous in $U^{0}$ and $x_{0} \in K$. Consequently, $\varphi$ is continuous at $x_{0} \in K$.

Let $x_{0} \in \mathbb{R}^{2} \backslash \bigcup_{k \in \mathbb{Z}}$ int $U^{k}$. There exists an $m \in \mathbb{Z}$ such that $x_{0} \in U^{m} \backslash$ int $U^{m}=f^{m+1}[K]$. Let $V$ be a neighbourhood of $x_{0}$ such that $V \cap f^{m}[K]=\emptyset$ and $V \cap f^{m+2}[K]=\emptyset$ (proceed as in the proof of the existence of $R$ satisfying (18)). Note that $V \subset U^{m} \cup \operatorname{int} U^{m+1}$, thus $f^{-m-1}[V] \subset U^{-1} \cup \operatorname{int} U^{0}$ and $f^{-m-1}[V]$ is a neighbourhood of $f^{-m-1}\left(x_{0}\right) \in K$.

Take any sequence $\left\{x_{k}\right\}$ in $V$ such that $x_{k} \rightarrow x_{0}$. Then $f^{-m-1}\left(x_{k}\right) \rightarrow$ $f^{-m-1}\left(x_{0}\right)$. Since $\varphi$ is continuous on $K$ and $f^{-m-1}\left(x_{0}\right) \in K$, we have $\varphi\left[f^{-m-1}\left(x_{k}\right)\right] \rightarrow \varphi\left[f^{-m-1}\left(x_{0}\right)\right]$. From (17) we have

$$
\varphi\left(x_{0}\right)=\varphi_{0}\left[f^{-m-1}\left(x_{0}\right)\right]+(m+1) a
$$

and

$$
\varphi\left(x_{k}\right)=\varphi_{0}\left[f^{-m-1}\left(x_{k}\right)\right]+(m+1) a \quad \text { for } k \in \mathbb{N} .
$$

Thus $\varphi\left(x_{k}\right) \rightarrow \varphi\left(x_{0}\right)$. Consequently, $\varphi$ is continuous on the plane.

Now assume, in addition, that $\varphi$ is one-to-one in $U^{0} \cup K$ and $\varphi_{0}(x)+n a \notin$ $\varphi_{0}\left[U^{0}\right]$ for all $x \in U^{0}$ and $n \in \mathbb{Z} \backslash\{0\}$. We show that $\varphi$ is one-to-one in the plane. Suppose $x, y \in \mathbb{R}^{2}$ and $\varphi(x)=\varphi(y)$. By (4), $x \in U^{k}$ and $y \in U^{l}$ for some $k, l \in \mathbb{Z}$. From (17) it follows that

$$
\varphi(x)=\varphi_{0}\left[f^{-k}(x)\right]+k a, \quad \varphi(y)=\varphi_{0}\left[f^{-l}(y)\right]+l a .
$$

Therefore

$$
\varphi_{0}\left[f^{-k}(x)\right]=\varphi_{0}\left[f^{-l}(y)\right]+(l-k) a .
$$

Suppose that $l-k \neq 0$. Then $\varphi_{0}\left[f^{-l}(y)\right]+(l-k) a \notin \varphi_{0}\left[U^{0}\right]$, since $f^{-l}(y) \in$ $U^{0}$. Hence $\varphi_{0}\left[f^{-k}(x)\right] \notin \varphi_{0}\left[U^{0}\right]$, which is a contradiction, since $f^{-k}(x) \in U^{0}$. Thus $l=k$, and consequently $x=y$. Note that $\varphi$, being a continuous oneto-one mapping of $\mathbb{R}^{2}$ into $\mathbb{R}^{2}$, is a homeomorphism (see [3], p. 186).

Now we show (c). Note that $\varphi[K]+a=\varphi[f[K]]$. Moreover, as $D_{\gamma} \cap$ $\varphi_{0}[K] \neq \emptyset$, we have $D_{\gamma} \cap \varphi_{0}[f[K]] \neq \emptyset$. Since $\left.\varphi\right|_{\text {int } U^{0}}$ is continuous and oneto-one, it is a homeomorphism and $\varphi\left[\operatorname{int} U^{0}\right]$ is a region. Moreover, $\varphi(x) \notin$ $\varphi\left[\operatorname{int} U^{0}\right]$, for every $x \in K \cup f[K]$, since $\varphi$ is one-to-one in $U^{0} \cup K$. Hence each $y \in \varphi[K] \cup \varphi[f[K]]$ is a boundary point of $\varphi\left[\right.$ int $\left.U^{0}\right]$, since $\varphi_{0}$ is continuous on $U^{0} \cup K$. Therefore $\varphi\left[\right.$ int $\left.U^{0}\right] \subset N^{0}$, because $\varphi_{0}[K] \cap D_{\gamma} \neq \emptyset$ and $\varphi_{0}[f[K]] \cap$ $D_{\gamma} \neq \emptyset$ for $\gamma \in \mathbb{R}$ and $\varphi_{0}[K]$ is a line. 
Let $x, y \in \mathbb{R}^{2}$. Then $x \in U^{k}$ and $y \in U^{l}$ for some $k, l \in \mathbb{Z}$. Assume that $\varphi(x)=\varphi(y)$. Then

$$
\varphi_{0}\left[f^{-k}(x)\right]=\varphi_{0}\left[f^{-l}(y)\right]+(l-k) a .
$$

Since $\varphi_{0}\left[U^{0}\right] \subset N^{0} \cup \varphi_{0}[f[K]]$, we have $l-k=0$, whence $x=y$. Thus $\varphi$ is continuous and one-to-one, and hence a homeomorphism.

Assume, in addition, that $\varphi_{0}\left[\operatorname{int} U^{0}\right]=N^{0}$. Put $W^{0}:=N^{0} \cup(\varphi[K]+a)$. Then $W^{0}=\varphi\left[U^{0}\right]$. Let $y \in \mathbb{R}^{2}$. Then there exists an $n \in \mathbb{Z}$ such that $y-n a \in W^{0}$. Take an $x \in U^{0}$ such that $\varphi(x)=y-n a$. Then by (1),

$$
\varphi\left[f^{n}(x)\right]=\varphi(x)+n a=y .
$$

Thus $\varphi\left[\mathbb{R}^{2}\right]=\mathbb{R}^{2}$. Consequently, $\varphi$ is a homeomorphism of $\mathbb{R}^{2}$ onto itself satisfying (1). This completes the proof.

Obviously, we also have the following

Remark 1. Let $f$ be a free mapping of the plane onto itself and let $a=\left(a_{1}, a_{2}\right) \in \mathbb{R}^{2} \backslash\{(0,0)\}$. Assume that condition (B) is satisfied. Let $\varphi$ be any homeomorphic solution of equation (1). Let $\varphi_{0}:=\left.\varphi\right|_{U^{0} \cup K}$. Then

(a) $\varphi_{0}$ is one-to-one and $\varphi_{0}\left[U^{0}\right] \cap\left(\varphi_{0}\left[U^{0}\right]+k a\right)=\emptyset$ for $k \in \mathbb{Z} \backslash\{0\}$;

(b) if $\varphi$ is a homeomorphism of $\mathbb{R}^{2}$ onto itself, then $\varphi_{0}$ is one-to-one, $\varphi_{0}[K]$ is a line, $\varphi_{0}[K] \cap D_{\gamma} \neq \emptyset$ for $\gamma \in \mathbb{R}$, and $\varphi_{0}\left[\operatorname{int} U^{0}\right]=N^{0}$, where $D_{\gamma}$ and $N^{0}$ are as in the statement of Theorem 2.

From part (d) of Theorem 2 we have

COROllary 1. If $f$ is a free mapping, then (B) implies (A).

From Lemma 2, Corollary 1 and the fact that $(\mathrm{A})$ implies $\left(\mathrm{A}^{\prime}\right)$ we have

Corollary 2. Let $f$ be a free mapping. Then conditions $(\mathrm{A}),\left(\mathrm{A}^{\prime}\right)$ and (B) are equivalent.

Now we are going to prove

Lemma 3. Let $f$ be a free mapping. Then (A) implies (C).

Proof. Put $L:=\left\{\left(x_{1}, x_{2}\right) \in \mathbb{R}^{2}: x_{1}=0\right\}$, and $D_{\alpha}:=\left\{\left(x_{1}, x_{2}\right) \in \mathbb{R}^{2}:\right.$ $\left.x_{2}=\alpha\right\}$ for $\alpha \in \mathbb{R}$. Let $K:=\varphi^{-1}[L]$ and $C_{\alpha}:=\varphi^{-1}\left[D_{\alpha}\right]$ for $\alpha \in \mathbb{R}$, where $\varphi$ is a homeomorphism satisfying $\varphi \circ f=T \circ \varphi$ with $T\left(x_{1}, x_{2}\right)=\left(x_{1}+1, x_{2}\right)$ for $x_{1}, x_{2} \in \mathbb{R}$. Let $I:=\mathbb{R}$. Since $f \circ \varphi^{-1}=\varphi^{-1} \circ T$ and $T\left[D_{\alpha}\right]=D_{\alpha}$ for $\alpha \in \mathbb{R}$, we have

$$
f\left[C_{\alpha}\right]=\varphi^{-1}\left[T\left[D_{\alpha}\right]\right]=\varphi^{-1}\left[D_{\alpha}\right]=C_{\alpha} \quad \text { for } \alpha \in \mathbb{R} .
$$

Moreover, note that

$$
\begin{gathered}
C_{\alpha} \cap C_{\beta}=\varphi^{-1}\left[D_{\alpha} \cap D_{\beta}\right]=\emptyset \quad \text { for } \alpha, \beta \in \mathbb{R}, \alpha \neq \beta, \\
\bigcup_{\alpha \in \mathbb{R}} C_{\alpha}=\varphi^{-1}\left[\bigcup_{\alpha \in \mathbb{R}} D_{\alpha}\right]=\varphi^{-1}\left[\mathbb{R}^{2}\right]=\mathbb{R}^{2}
\end{gathered}
$$


and

$$
\operatorname{card}\left(K \cap C_{\alpha}\right)=\operatorname{card} \varphi^{-1}\left[L \cap D_{\alpha}\right]=\operatorname{card}\left(L \cap D_{\alpha}\right)=1
$$

for $\alpha \in \mathbb{R}$. This completes the proof.

Theorem 3. Let $f$ be a free mapping. Then condition (C) implies (B).

P r o of. Suppose that (C) holds.

1. First, we show that for the line $K$ which appears in (C), $K \cap f[K]=\emptyset$.

Suppose $x_{0} \in K \cap f[K]$. On account of (8), $x_{0} \in C_{\alpha}$ for some $\alpha \in I$. By (5) we get $f^{-1}\left(x_{0}\right) \in C_{\alpha}$, and clearly $f^{-1}\left(x_{0}\right) \in K$. Since card $\left(K \cap C_{\alpha}\right)=$ $1, x_{0}=f^{-1}\left(x_{0}\right)$. Hence $x_{0}$ is a fixed point of $f$, a contradiction.

2. Now we prove that

$$
\operatorname{card}\left(f^{n}[K] \cap C_{\alpha}\right)=1 \quad \text { for } \alpha \in I \text { and } n \in \mathbb{Z} .
$$

Fix any $\alpha \in I$. Let $n \in \mathbb{Z} \backslash\{0\}$. Take $x_{0} \in K \cap C_{\alpha}$. By (5),

$$
f^{n}\left(x_{0}\right) \in f^{n}[K] \cap C_{\alpha} .
$$

Suppose there exist $y_{1}, y_{2} \in f^{n}[K] \cap C_{\alpha}$ such that $y_{1} \neq y_{2}$. Then $f^{-n}\left(y_{1}\right)$, $f^{-n}\left(y_{2}\right) \in K \cap C_{\alpha}$ and $f^{-n}\left(y_{1}\right) \neq f^{-n}\left(y_{2}\right)$, which contradicts (7).

3 . Let $x \in C_{\alpha}$. We now prove that, for every $n \in \mathbb{Z}, f^{n+1}(x)$ lies between $f^{n}(x)$ and $f^{n+2}(x)$ on the curve $C_{\alpha}$. For any $x, y \in C_{\alpha}$ denote by $\langle x, y\rangle$ the segment of $C_{\alpha}$ with endpoints $x, y$. Let $(x, y):=\langle x, y\rangle \backslash\{x, y\}$.

Let $n \in \mathbb{Z}$. If $f^{n+2}(x) \in\left(f^{n}(x), f^{n+1}(x)\right) \subset C_{\alpha}$, then

$$
f\left(\left\langle f^{n}(x), f^{n+1}(x)\right\rangle\right)=\left\langle f^{n+2}(x), f^{n+1}(x)\right\rangle \subset\left\langle f^{n}(x), f^{n+1}(x)\right\rangle .
$$

Hence by Brouwer's Theorem $f$ has a fixed point, which is impossible. Similarly, if $f^{n}(x) \in\left(f^{n+1}(x), f^{n+2}(x)\right) \subset C_{\alpha}$, then

$$
f^{-1}\left(\left\langle f^{n+1}(x), f^{n+2}(x)\right\rangle\right)=\left\langle f^{n+1}(x), f^{n}(x)\right\rangle \subset\left\langle f^{n+1}(x), f^{n+2}(x)\right\rangle .
$$

Hence $f^{-1}$ has a fixed point, contradiction again. Thus

$$
f^{n+1}(x) \in\left(f^{n}(x), f^{n+2}(x)\right) .
$$

4. Now we show that (3) holds. Since, $f^{n}[K]$ is a line for all $n \in \mathbb{Z}$, $\mathbb{R}^{2} \backslash f^{n}[K]$ consists of two unbounded regions, called the side domains of $f^{n}[K]$. Since $K \cap f[K]=\emptyset$, we have $f^{n}[K] \cap f^{n+1}[K]=\emptyset$ for all $n \in \mathbb{Z}$. For each $n \in \mathbb{Z}$, denote by $M^{n}$ the strip between the lines $f^{n}[K]$ and $f^{n+1}[K]$. Let $M_{+}^{n}$ be the side domain of $f^{n+1}[K]$ which does not contain the line $f^{n}[K]$, and $M_{-}^{n}$ the side domain of $f^{n}[K]$ which does not contain $f^{n+1}[K]$. Then

$$
M_{-}^{n} \cup f^{n}[K] \cup M^{n} \cup f^{n+1}[K] \cup M_{+}^{n}=\mathbb{R}^{2} \quad \text { for } n \in \mathbb{Z} .
$$

Now we show that $f^{n+2}[K] \subset M_{+}^{n}$ for $n \in \mathbb{Z}$. Suppose otherwise. Then for some $n \in \mathbb{Z}$,

$$
f^{n+2}[K] \subset M_{-}^{n} \cup f^{n}[K] \cup M^{n},
$$


since $f^{n+2}[K] \cap f^{n+1}[K]=\emptyset$. Take any $x_{0} \in K$. By (8), $x_{0} \in C_{\alpha}$ for some $\alpha \in I$. By (5), $f^{n}\left(x_{0}\right) \in C_{\alpha}$ for all $n \in \mathbb{Z}$. From (20) we obtain $f^{n+2}\left(x_{0}\right) \in M_{-}^{n} \cup f^{n}[K] \cup M^{n}$. Thus by (19),

$$
C_{\alpha} \subset M_{-}^{n} \cup f^{n}[K] \cup M^{n} \cup\left\{f^{n+1}\left(x_{0}\right)\right\},
$$

since

$$
f^{n}\left(x_{0}\right), f^{n+2}\left(x_{0}\right) \in M_{-}^{n} \cup f^{n}[K] \cup M^{n}, \quad f^{n+1}[K] \cap C_{\alpha}=\left\{f^{n+1}\left(x_{0}\right)\right\}
$$

and $C_{\alpha}$ has no self-intersections. Consequently, we have shown that for each $\alpha \in I$,

$$
C_{\alpha} \subset M_{-}^{n} \cup f^{n}[K] \cup M^{n} \cup f^{n+1}[K]=\mathbb{R}^{2} \backslash M_{+}^{n},
$$

which contradicts (8). Thus $f^{n+2}[K] \subset M_{+}^{n}$ for all $n \in \mathbb{Z}$. Hence $M^{n}, n \in \mathbb{Z}$, are mutually disjoint and $f^{n}[K] \cap K=\emptyset$ for $n \in \mathbb{Z} \backslash\{0\}$. Since $f$ is a homeomorphism, we have

$$
f^{n}\left[M^{0}\right]=M^{n} \quad \text { for } n \in \mathbb{Z} .
$$

Thus $f^{n}\left[M^{0}\right] \cap M^{0}=\emptyset$ for $n \in \mathbb{Z} \backslash\{0\}$. Moreover, for every $n \in \mathbb{Z} \backslash\{0\}$,

$$
f^{n}\left[M^{0} \cup f[K]\right] \cap\left(M^{0} \cup f[K]\right)=\left(f^{n}\left[M^{0}\right] \cup f^{n+1}[K]\right) \cap\left(M^{0} \cup f[K]\right)=\emptyset .
$$

Thus, for all $n \in \mathbb{Z} \backslash\{0\}, f^{n}\left[U^{0}\right] \cap U^{0}=\emptyset$, where $U^{0}=M^{0} \cup f[K]$.

5. To complete the proof we show that

$$
\bigcup_{n \in \mathbb{Z}} f^{n}\left[U^{0}\right]=\mathbb{R}^{2}
$$

For each $\alpha \in I$ let $K \cap C_{\alpha}=:\left\{x_{\alpha}\right\}$ and $C_{\alpha}^{0}=\left(x_{\alpha}, f\left(x_{\alpha}\right)\right) \subset C_{\alpha}$. First, we show that $\bigcup_{\alpha \in I} C_{\alpha}^{0}=M^{0}$.

Suppose that $x_{0} \in C_{\alpha}^{0}$ and $x_{0} \notin M^{0}$. Then $C_{\alpha}^{0}$ has either a common point with $K$ different from $x_{\alpha}$ or a common point with $f[K]$ different from $f\left(x_{\alpha}\right)$, which is impossible.

For each $\alpha \in I$ denote by $C_{\alpha}^{0+}$ the set of all $x \in C_{\alpha}$ such that $f\left(x_{\alpha}\right) \in$ $\left(x_{\alpha}, x\right) \subset C_{\alpha}$, and by $C_{\alpha}^{0-}$ the set of all $x \in C_{\alpha}$ such that $x_{\alpha} \in\left(x, f\left(x_{\alpha}\right)\right)$ $\subset C_{\alpha}$.

Take any $x_{0} \in M^{0}$. Then $x_{0} \in C_{\alpha}$ for some $\alpha \in I$. Suppose that $x_{0} \in$ $C_{\alpha}^{0+}$. Since $\operatorname{card}\left(C_{\alpha} \cap f[K]\right)=1$ and $f\left(x_{\alpha}\right) \in C_{\alpha} \cap f[K]$, we have $C_{\alpha}^{0+} \cap$ $f[K]=\emptyset$. Hence $C_{\alpha}^{0+}$ is contained either in $M_{+}^{0}$ or in $M_{-}^{0} \cup K \cup M^{0}$. Since $f^{2}\left(x_{0}\right) \in C_{\alpha}^{0+} \cap M_{+}^{0}$, we have $C_{\alpha}^{0+} \subset M_{+}^{0}$, whence $x_{0} \in M_{+}^{0}$, but this is impossible, since $x_{0} \in M^{0}$.

Now suppose $x_{0} \in C_{\alpha}^{0-}$. Since $\operatorname{card}\left(C_{\alpha} \cap K\right)=1$ and $x_{\alpha} \in C_{\alpha} \cap K$, we have $C_{\alpha}^{0-} \cap K=\emptyset$. Hence $C_{\alpha}^{0-}$ is contained either in $M_{-}^{0}$ or in $M^{0} \cup f[K] \cup$ $M_{+}^{0}$. Since $f^{-1}\left(x_{0}\right) \in C_{\alpha}^{0-} \cap M_{-}^{0}$, we have $C_{\alpha}^{0-} \subset M_{-}^{0}$, whence $x_{0} \in M_{-}^{0}$, and this is also impossible. Consequently,

$$
\bigcup_{\alpha \in I} C_{\alpha}^{0}=M^{0} \text {. }
$$


For every $\alpha \in I$ and every $n \in \mathbb{Z}$, let $C_{\alpha}^{n}:=\left(f^{n}\left(x_{\alpha}\right), f^{n+1}\left(x_{\alpha}\right)\right) \subset C_{\alpha}$. Since $f$ is a homeomorphism, we have by (5),

$$
C_{\alpha}^{n}=f^{n}\left[C_{\alpha}^{0}\right] \quad \text { for } \alpha \in I \text { and } n \in \mathbb{Z} .
$$

Hence, for all $n \in \mathbb{Z}$, we get by (21) and (22),

$$
M^{n}=f^{n}\left[M^{0}\right]=\bigcup_{\alpha \in I} f^{n}\left[C_{\alpha}^{0}\right]=\bigcup_{\alpha \in I} C_{\alpha}^{n} .
$$

Let $x_{0} \in \mathbb{R}^{2}$. If there exists an $n \in \mathbb{Z}$ such that $x_{0} \in f^{n}[K]$, then $x_{0} \in f^{n-1}\left[U^{0}\right]$. Now assume that $x_{0} \in \mathbb{R}^{2} \backslash \bigcup_{n \in \mathbb{Z}} f^{n}[K]$. Then $x_{0} \in C_{\alpha}$ for some $\alpha \in I$. Since $f^{n}\left(x_{\alpha}\right) \rightarrow \infty$ as $n \rightarrow \mp \infty$ (see [1], Prop. 1.2), there is an $n \in \mathbb{Z}$ such that $x_{0} \in C_{\alpha}^{n}$. Hence by (22) and (23),

$$
x_{0} \in f^{n}\left[C_{\alpha}^{0}\right] \subset f^{n}\left[M^{0}\right] \subset f^{n}\left[U^{0}\right] .
$$

Consequently, $\mathbb{R}^{2}=\bigcup_{n \in \mathbb{Z}} f^{n}\left[U^{0}\right]$. This completes the proof.

Note that Theorem 1 is a consequence of Corollary 2, Lemma 3 and Theorem 3.

Moreover, from the proof of Theorem 3 we have the following

Corollary 3. Let $f$ be a free mapping. Let $K$ be a line on the plane. If $K$ satisfies condition $(\mathrm{C})$, then it also satisfies (B).

3. In this section we study diffeomorphic solutions of equation (1). First we quote the following

Lemma 4 (see [5]). If the functions $f$ and $\varphi$ are of class $C^{p}(p>0)$ in a region $U \subset \mathbb{R}^{n}$ such that $f[U] \subset U$, then for $x \in U$,

$$
\frac{\partial^{q}}{\partial x_{i_{1}} \ldots \partial x_{i_{q}}} \varphi[f(x)]=\sum_{k=1}^{q} \sum_{j_{1}, \ldots, j_{k}=1}^{n} b_{i_{1} \ldots i_{q}}^{j_{1} \ldots j_{k}}(x) \varphi_{j_{1} \ldots j_{k}}[f(x)],
$$

$q=1, \ldots, p$, where

$$
\varphi_{i_{1} \ldots i_{k}}(x)=\frac{\partial^{k}}{\partial x_{i_{1}} \ldots \partial_{i_{k}}} \varphi(x)
$$

$b_{i_{1} \ldots i_{q}}^{j_{1} \ldots j_{k}}(x)$ may be expressed by means of sums and products of $a_{i}^{j}(x), \ldots$ $\ldots, a_{i_{1}, \ldots, i_{q-k+1}}^{j}(x), a_{i_{1}, \ldots, i_{k}}^{j}(x)=\frac{\partial^{k}}{\partial x_{i_{1}} \ldots \partial x_{i_{k}}} f_{j}(x), k=1, \ldots, p$, and $f=$ $\left(f_{1}, \ldots, f_{n}\right)$. Consequently, $b_{i_{1} \ldots i_{q}}^{j_{1} \ldots j_{k}}$ are of class $C^{p-q+k-1}$. In particular,

$$
b_{i_{1} \ldots i_{q}}^{j_{1} \ldots j_{q}}(x)=a_{i_{1}}^{j_{1}}(x) \cdot \ldots \cdot a_{i_{q}}^{j_{q}}(x) .
$$

Now let $f$ be a free mapping. Assume that condition (B) is satisfied. 
Definition (see [5]) . Let $\psi$ be a continuous function defined in $U^{0} \cup K$, $p$ times continuously differentiable in $\operatorname{int} U^{0}$. We write

$$
\psi_{i_{1} \ldots i_{k}}\left(x_{0}\right)=\lim _{\substack{x \rightarrow x_{0} \\ x \in \operatorname{int} U^{0}}} \frac{\partial^{k}}{\partial x_{i_{1}} \ldots \partial x_{i_{k}}} \psi(x), \quad k=1, \ldots, p,
$$

for $x_{0} \in K \cup f[K]$ (provided this limit exists). The function $\psi$ is said to be of class $C^{p}$ in $U^{0} \cup K$ if all the functions $\psi, \psi_{i}, \ldots, \psi_{i_{1} \ldots i_{p}}$ are continuous in $U^{0} \cup K$.

All diffeomorphic solutions of equation (1) having positive Jacobian can be obtained from the following

THEOREM 4. Let $f$ be a free $C^{p}$ mapping of the plane having positive Jacobian at every $x \in \mathbb{R}^{2}$ and let $a=\left(a_{1}, a_{2}\right) \in \mathbb{R}^{2} \backslash\{(0,0)\}$. Assume that condition (B) is satisfied. Let $\psi$ be a $C^{p}$ function from $U^{0} \cup K$ into the plane which satisfies

$$
\begin{gathered}
\psi[f(x)]=\psi(x)+a \quad \text { for } x \in K, \\
\sum_{k=1}^{q} \sum_{j_{1}, \ldots, j_{k}=1}^{2} b_{i_{1} \ldots i_{q}}^{j_{1} \ldots j_{k}}(x) \psi_{j_{1} \ldots j_{k}}[f(x)]=\psi_{i_{1} \ldots i_{q}}(x)
\end{gathered}
$$

for $x \in K, q=1, \ldots, p, i_{1}, \ldots, i_{q}=1,2$, where the functions $b_{i_{1} \ldots i_{q}}^{j_{1} \ldots j_{k}}$ are those occurring in Lemma 4. Then there exists a unique solution $\varphi$ of equation (1) such that

$$
\varphi(x)=\psi(x) \quad \text { for } x \in U^{0} \cup K .
$$

This solution is of class $C^{p}$ in the plane. Moreover, if $\psi$ is one-to-one, the Jacobian, $\operatorname{jac}_{x} \psi$, is positive for $x \in \operatorname{int} U^{0}$, and $\operatorname{det}\left[\psi_{1}(x), \psi_{2}(x)\right]>0$ for $x \in K \cup f[K]$, and either

$$
\psi\left[U^{0}\right] \cap\left(\psi\left[U^{0}\right]+k a\right)=\emptyset \quad \text { for } k \in \mathbb{Z} \backslash\{0\}
$$

or

$$
\psi[K] \cap D_{\gamma} \neq \emptyset \quad \text { for } \gamma \in \mathbb{R} \text { and } \psi[K] \text { is a line, }
$$

where $D_{\gamma}=\left\{\left(x_{1}, x_{2}\right) \in \mathbb{R}^{2}: a_{2} x_{1}-a_{1} x_{2}=\gamma\right\}$, then $\varphi$ is an orientation preserving diffeomorphism of class $C^{p}$ having positive Jacobian.

Proof. Define $\varphi$ by setting

$$
\varphi(x)=\psi\left[f^{-k}(x)\right]+k a, \quad x \in U^{k}, k \in \mathbb{Z},
$$

where $U^{k}=f^{k}\left[U^{0}\right]$. For $p=0$ we have Theorem 1. Let $p>0$. From (24) it follows that $\varphi$ is of class $C^{p}$ in $\bigcup_{k \in \mathbb{Z}}$ int $U^{k}$.

Let $x_{0} \in K$. Then there exists an open disc $R$ with centre at $x_{0}$ such that $R \cap f^{-1}[K]=\emptyset$ and $R \cap f[K]=\emptyset$ (see the proof of Theorem 2). The proof of $\varphi$ being $C^{p}$ in $R$ runs in the same way as that of Theorem 3.1 in [5], part 2. 
Let $x_{0} \in \mathbb{R}^{2} \backslash \bigcup_{k \in \mathbb{Z}}$ int $U^{k}$. There is an $m \in \mathbb{Z}$ such that $f^{-m-1}\left(x_{0}\right) \in K$. We have already proved that $\varphi$ is $C^{p}$ in a neighbourhood $R$ of $f^{-m-1}\left(x_{0}\right)$. The function $f^{m+1}$ is a $C^{p}$ map of $R$ onto a neighbourhood $f^{m+1}[R]$ of $x_{0}$. Since $\varphi$ is a solution of (1), we have

$$
\varphi\left(x_{0}\right)=\varphi\left[f^{-m-1}\left(x_{0}\right)\right]+(m+1) a .
$$

Hence $\varphi$ is $C^{p}$ in $f^{m+1}[R]$.

Now assume, in addition, that $\psi$ is one-to-one, $\psi(x)+k a \notin \psi\left[U^{0}\right]$ for $x \in U^{0}$ and $k \in \mathbb{Z} \backslash\{0\}$ [or $\psi[K] \cap D_{\gamma} \neq \emptyset$ for all $\gamma \in \mathbb{R}$ and $\psi[K]$ is a line], $\operatorname{jac}_{x} \psi>0$ for $x \in \operatorname{int} U^{0}$ and $\operatorname{det}\left[\psi_{1}(x), \psi_{2}(x)\right]>0$ for $x \in K \cup f[K]$. On account of Theorem 2, $\varphi$ is a homeomorphism.

If $x \in U^{0}$, then $\operatorname{jac}_{x} \varphi=\operatorname{jac}_{x} \psi>0$. If $x \in f[K]$, then $\operatorname{jac}_{x} \varphi=$ $\operatorname{det}\left[\psi_{1}(x), \psi_{2}(x)\right]>0$, since $\left(\partial \varphi / \partial x_{1}\right)(x)=\psi_{1}(x)$ and $\left(\partial \varphi / \partial x_{2}\right)(x)=\psi_{2}(x)$ for $x \in f[K]$. Thus $\operatorname{jac}_{x} \varphi>0$ for $x \in U^{0}$.

Let $x \in \mathbb{R}^{2}$. Then $x \in f^{n}\left[U^{0}\right]$ for some $n \in \mathbb{Z}$. Since $\varphi(x)=\varphi\left[f^{-n}(x)\right]+$ $n a$, we have

$$
\operatorname{jac}_{x} \varphi=\operatorname{jac}_{f^{-n}(x)} \varphi \cdot \operatorname{jac}_{x} f^{-n} .
$$

Hence $\operatorname{jac}_{x} \varphi>0$, since $f^{-n}(x) \in U^{0}$ and $\operatorname{jac}_{x} f^{-n}>0$. Thus $\varphi$ preserves orientation. Since $\varphi$ is invertible and of class $C^{p}$, and $\operatorname{jac}_{x} \varphi \neq 0$ for $x \in \mathbb{R}^{2}$, $\varphi^{-1}$ is $C^{p}$ (see e.g. [6], p. 205). This completes the proof.

\section{References}

[1] S. A. Andrea, On homeomorphisms of the plane which have no fixed points, Abh. Math. Sem. Hamburg 30 (1967), 61-74.

[2] - , The plane is not compactly generated by a free mapping, Trans. Amer. Math. Soc. 151 (1970), 481-498.

[3] R. Engelking and K. Sieklucki, Topology. A Geometric Approach, Sigma Ser. Pure Math. 4, Heldermann, Berlin 1992.

[4] T. Homma and H. Terasaka, On the structure of the plane translation of Brouwer, Osaka Math. J. 5 (1953), 233-266.

[5] M. Kuczma, On the Schröder equation, Rozprawy Mat. 34 (1963).

[6] R. Sikorski, Advanced Calculus. Functions of Several Variables, Monograf. Mat. 52, PWN, Warszawa 1969.

[7] M. C. Zdun, On continuous iteration groups of fixed-point free mapping in $\mathbb{R}^{2}$ space, in: Proc. European Conference on Iteration Theory, Batschuns 1989, World Scientific, Singapore 1991, 362-368.

INSTITUTE OF MATHEMATICS

PEDAGOGICAL UNIVERSITY OF CRACOW

PODCHORĄŻYCH 2

30-084 KRAKÓW, POLAND 